\title{
Mucoepidermoid Carcinoma of Palatal Minor Salivary Glands with Intracranial Extension: A Case Report and Literature Review
}

\author{
Rimal Hanif Dossani ${ }^{1}$ Hesam Akbarian-Tefaghi ${ }^{1}$ Lori Lemonnier ${ }^{2}$ Vikas Mehta ${ }^{2}$ Jamie A. Jacobsohn ${ }^{3}$ \\ Bharat Guthikonda ${ }^{1}$ \\ ${ }^{1}$ Department of Neurosurgery, LSUHSC Shreveport, Shreveport, \\ Louisiana, United States \\ ${ }^{2}$ Department of Otolaryngology/Head and Neck Surgery, LSUHSC \\ Shreveport, Shreveport, Louisiana, United States \\ ${ }^{3}$ Department of Pathology, LSUHSC Shreveport, Shreveport, \\ Louisiana, United States \\ J Neurol Surg Rep 2016;77:e156-e159.

\begin{abstract}
Address for correspondence Bharat Guthikonda, MD, Department of Neurosurgery, LSU HSC Shreveport, Shreveport, LA 71130-3932, United States (e-mail: bguthi@lsuhsc.edu).
\end{abstract}

\author{
Abstract \\ Keywords \\ - mucoepidermoid \\ carcinoma \\ - intracranial extension \\ - trigeminal nerve \\ - perineural invasion
}

Mucoepidermoid carcinoma (MEC) is one of the most common malignant tumors of both major and minor salivary glands. Although there are reports of parotid MEC tumors extending intracranially via the facial nerve, intracranial extension from MEC originating from minor salivary glands in the palate has not previously been reported. This report presents a case of MEC arising from the minor salivary glands of the palate and extending into the middle fossa via the foramen rotundum with perineural invasion of the maxillary division of the trigeminal nerve. The patient received surgical intervention via a combined otolaryngology and neurosurgery approach to achieve gross total resection of the tumor. This was followed by adjuvant radiotherapy. The epidemiology, histopathology, and treatment of MEC originating from salivary glands are discussed.

\section{Introduction}

Mucoepidermoid carcinoma (MEC) is one of the most common malignant neoplasms of the salivary glands in both pediatric and adult populations. ${ }^{1}$ Among the major salivary glands, the most common site of MEC is the parotid gland. ${ }^{2}$ MEC tumors of parotid origin have been reported to extend intracranially to the temporal bone and cerebellopontine angle via the perineurium of the facial nerve. ${ }^{3}$ Perineural invasion is a sign of high-grade MEC, worse patient outcomes, and a need for more aggressive surgical intervention. ${ }^{4,5} \mathrm{MEC}$ of the minor salivary glands most commonly occurs in the oral cavity at the junction of the hard and soft palate. To date, intracranial extension of palatal salivary gland MEC has not been reported. Here, we report a case of an adult patient with MEC originating from the minor salivary glands of the nasopharyngeal portion of the soft palate, extending into the right

received

March 27, 2016 accepted after revision August 19, 2016 lateral wall of the nasopharynx, the pterygopalatine and infratemporal fossae, and reaching the anteromedial aspect of the middle fossa to involve the anterior temporal pole. Intracranial extension of the palatal MEC tumor likely occurred from the perineural invasion of the infraorbital nerve with the retrograde involvement of the maxillary branch of the trigeminal nerve at the foramen rotundum.

\section{Case Report}

The patient is a 53-year-old Caucasian male with a 2-month history of nasal obstruction and right-sided hearing loss. On physical examination, there was a visible serous effusion behind the right tympanic membrane. A computed tomography (CT) scan with contrast indicated the presence of a poorly defined soft tissue mass in the right posterior naso/oropharynx. Patient evaluation by an audiologist showed a moderate-to-severe
License terms

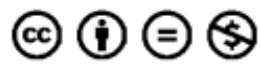




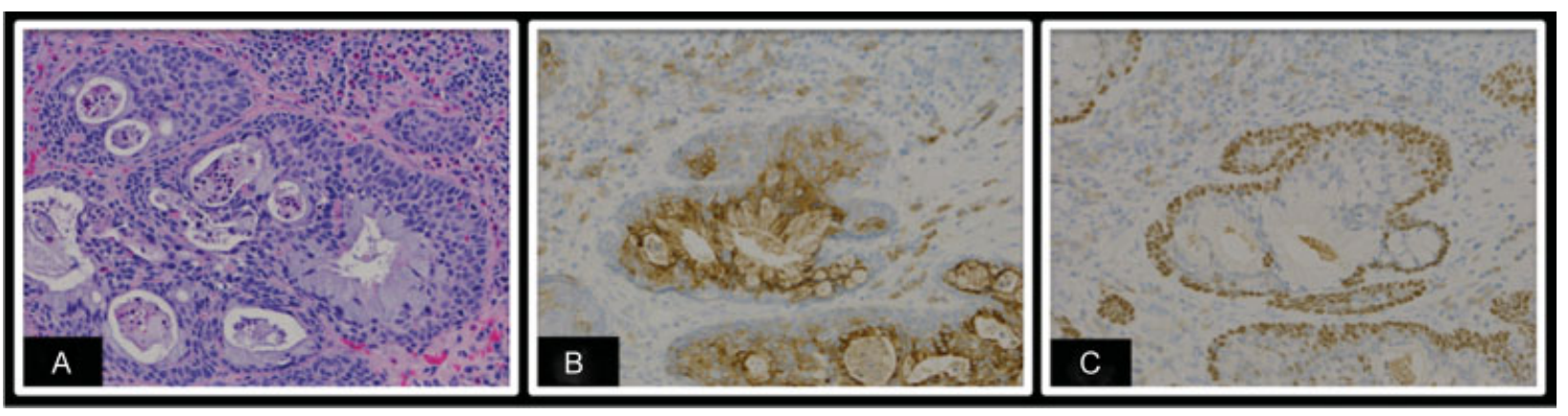

Fig. 1 (A) $\mathrm{H}^{+} \mathrm{E}$ : Mucoepidermoid carcinoma in the nasopharynx with tumor nests showing mucinous and squamous differentiation; (B) EMA: An epithelial membrane antigen antibody highlights tumor cells with glandular differentiation; (C) p63: A p63 antibody highlights tumor cells with squamous differentiation.

conductive hearing loss in the right ear with complete sparing of the hearing functions on the left side. Nasal endoscopy revealed an exophytic mass involving the right lateral nasopharyngeal wall and obstructing the eustachian tube.

An endoscopic incisional biopsy of the accessible portion of the right nasopharyngeal mass was interpreted as a high-grade MEC with cystic and solid nests of squamous and mucinous glandular cells. Moderate nuclear atypia, frequent mitosis (up to 20 per 10 high powered fields), areas of necrosis, and perineural invasion were seen ( $\boldsymbol{- F i g s .} 1$ and 2). During this procedure, the mass was noted to block the right eustachian tube posteriorly and to overlie the soft palate inferiorly. Magnetic resonance imaging (MRI) of the orbit and face demonstrated a mass arising from the nasopharynx extending into the infratemporal fossa and pterygopalatine region (-Fig. 3). MRI also showed the involvement of the maxillary branch of the trigeminal nerve, the cavernous sinus, and the anterior temporal pole in the middle cranial fossa.

The patient underwent joint resection of the tumor with otolaryngology and neurosurgery involvement. An endoscopic transnasal approach was used to resect the portion of the lesion involving the lateral nasal wall, nasal floor, nasal speculum, nasopharynx, sphenoid sinus, ethmoid sinus, pterygopalatine fossa, and infratemporal fossa. Within the pterygopalatine fossa,

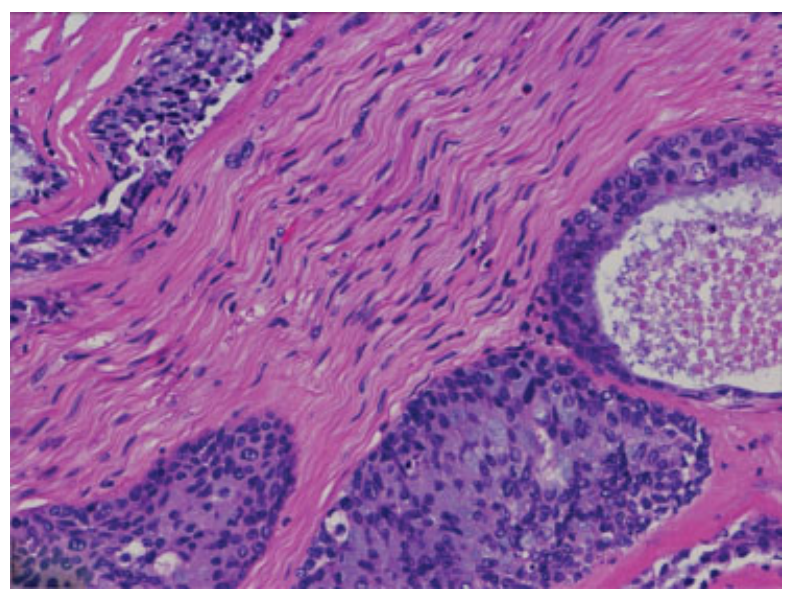

Fig. $2 \mathrm{H}^{+} \mathrm{E}$ : Mucoepidermoid carcinoma with perineural and endoneurial invasion of the $\mathrm{V} 2$ branch of the trigeminal nerve. the tumor was noted to be pedicled superiorly to the foramen rotundum. Following endoscopic endonasal tumor resection, transoral resection of the involved areas of the palate was performed. The soft palate and uvula were split in half to facilitate tumor resection from the nasal aspect of the soft palate. No tumor was visualized proximal to the junction of the hard and soft palate in the oral cavity.

The neurosurgery team performed a middle fossa craniotomy. Extradural dissection demonstrated the involvement of V2 division of the trigeminal nerve. The V1 and V3 nerves were also visualized and were within normal neural tissue inspection. The V2 branch that was infiltrated by tumor was sacrificed to the level of the foramen rotundum through the opening into the pterygopalatine fossa to unite with the endoscopic portion of the resection. A nasoseptal flap was used to repair the defect.

At 2-months follow-up, the patient was doing well without a cerebrospinal fluid leak or new neurological deficit and endoscopic biopsy showed no evidence of gross disease. His main postoperative complaint has been a burning sensation in the right V2 distribution, which is being managed with trigeminal nerve blocks. The patient underwent adjuvant radiotherapy with posttreatment imaging (positron emission tomography/CT and MRI) at 3 months postradiation completion showing complete remission.

\section{Discussion}

Intracranial extension of MEC is extremely rare. To date, only one case report in the literature has discussed cerebellopontine angle extension of parotid MEC arising from perineural invasion of the facial nerve. ${ }^{6}$ Our case report is the first to demonstrate intracranial extension along the maxillary division of the trigeminal nerve via the foramen rotundum in an MEC arising from a minor salivary gland of the palate. Our patient underwent gross total resection of high-grade MEC followed by radiation therapy and has been free of recurrence on most recent follow-up, 7 months after surgery.

MEC is one of the most common malignant neoplasms observed in the major and minor salivary glands. ${ }^{2}$ MEC most commonly affects the parotid gland among the major salivary glands. Approximately 450 to 750 minor salivary glands are present in the head and neck. ${ }^{5}$ These minor glands are most 


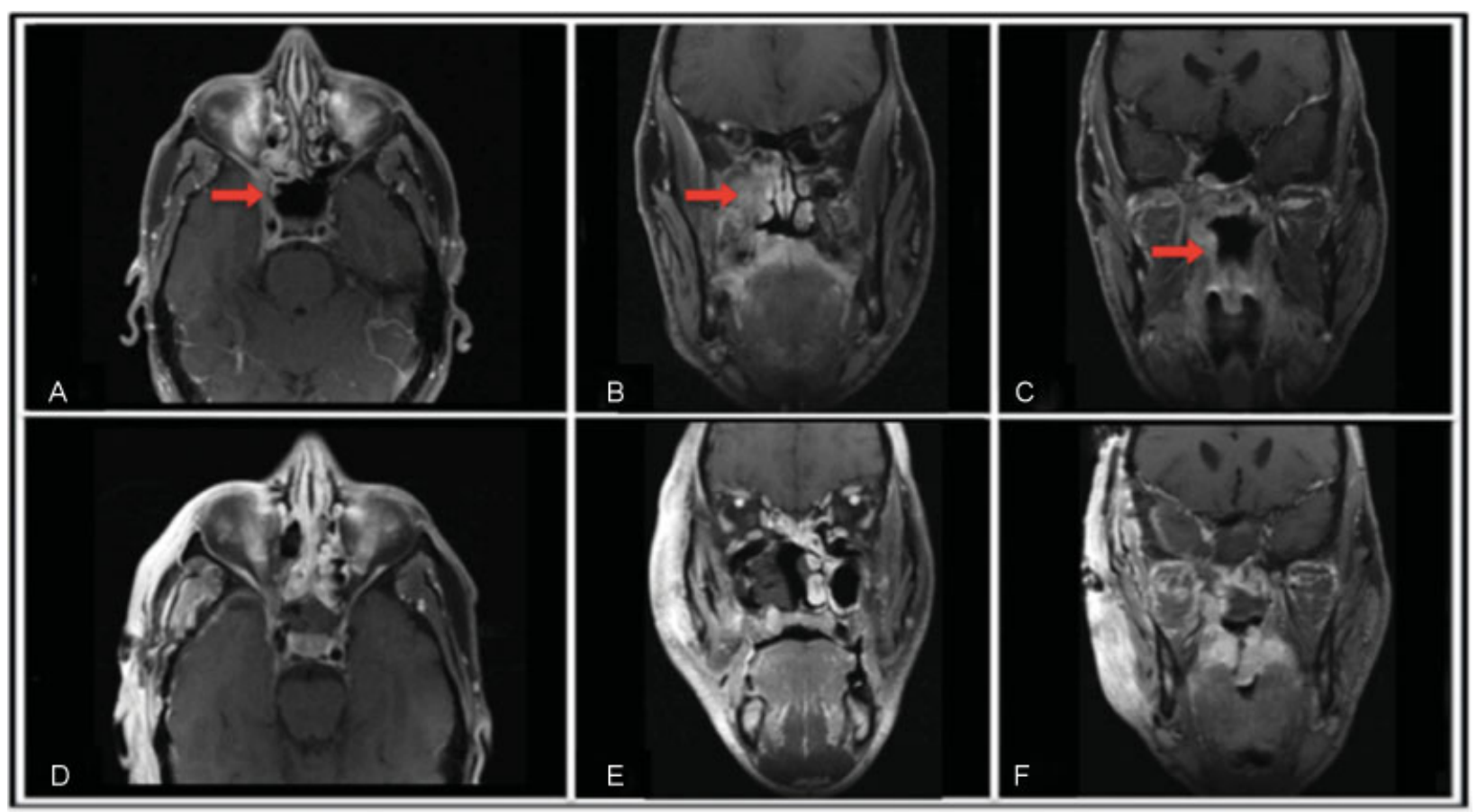

Fig. 3 Preoperative MRI show a large mass infiltrating the right lateral wall of the nasopharynx, extending to the pterygomaxillary and infratemporal fossa, and reaching the anteromedial aspect of the middle fossa to come in close contact with the temporal pole of the brain: (A) Pre-op axial, (B) Pre-op coronal-A, (C) Pre-op coronal-B. Post-operative MRI shows the resolution of the lesion: (D) Post-op axial, (E) Post-op coronalA, (F) Post-op coronal-B.

commonly situated at the junction of the hard and soft palate. Around 8 to $15 \%$ of salivary gland tumors arise in the palate and are reportedly malignant in 40 to $82 \%$ of patients. Minor salivary glands tumors are more likely to be malignant than tumors of major salivary glands. ${ }^{5}$ In one study of intraoral minor salivary gland neoplasms, MEC comprised $21 \%$ of all tumors and $48 \%$ of all malignant tumors. ${ }^{7}$

In the oral cavity, MEC presents as a fixed, rubbery, and painless mass. The most common symptom is swelling, followed by pain, ulceration, and discoloration. The World Health Organization defines MEC as "a malignant glandular epithelial carcinoma characterized by mucous, intermediate, and epithelial cells, with columnar, clear cell and oncocytoid features." ${ }^{8}$ The "intermediate cells" are difficult to characterize as their description varies in the literature. One review article describes intermediate cells as "nondescript" cells with a morphology that does not match a differentiated or recognized phenotype, such as mucous or squamoid. ${ }^{8}$

Determination of low-grade versus high-grade MEC is based on morphological descriptions of predominant cell types in the tumor. ${ }^{9}$ The low-grade type is characterized by $>50 \%$ mucinous cells while the high-grade type is characterized by a predominance of epidermoid cells with $<10 \%$ mucinous cells. In a study of 55 patients with long-term follow-up over a period of 30 years, 5 and 10-year survival rate are 92.4 and $90.1 \%$, respectively, regardless of histological classification. Accounting for histological classification, the study demonstrated a 10 -year survival rate of $96.7 \%$ in highly differentiated MEC as compared with $81.6 \%$ in poorly differentiated tumors. A database study involving 2,400 patients showed no difference in 5-year disease-specific survival between intermediate and low-grade MEC tumors (97.4 vs. $98.8 \%$, respectively). ${ }^{10}$

The first-line treatment for MEC is surgical resection with the goal of disease-free margins while minimizing morbidity. ${ }^{8}$ A retrospective review of MEC in major salivary glands demonstrated that, of the 234 patients, 208 received surgical therapy only, 22 received combined surgery and radiation therapy, and 2 underwent surgery followed by radiation and chemotherapy. ${ }^{2}$ This review also demonstrated that patients with positive surgical margins receiving adjuvant radiation had survival times comparable to those with complete surgical resection. The role of chemotherapy is not well defined. The Radiation Therapy Oncology Group (RTOG) is performing a phase II randomized control trial to determine whether the administration of cisplatin with postoperative radiotherapy improves outcomes in patients with high-risk salivary tumors, including MEC. ${ }^{8}$

\section{Conclusion}

We present the first report of a nasopharyngeal MEC arising from the minor salivary glands of the palate to have a perineural intracranial extension. The patient was successfully managed with surgical resection followed by radiation therapy. MECs of the minor salivary glands originating in the palate and extending to the brain require a multidisciplinary surgical approach to achieve gross total resection of both intra- and extracranial components. Close follow-up and adjuvant radiotherapy are advised to decrease the risk of tumor recurrence. 


\section{References}

1 Sultan I, Rodriguez-Galindo C, Al-Sharabati S, Guzzo M, Casanova M, Ferrari A. Salivary gland carcinomas in children and adolescents: a population-based study, with comparison to adult cases. Head Neck 2011;33(10):1476-1481

2 Goode RK, Auclair PL, Ellis GL. Mucoepidermoid carcinoma of the major salivary glands: clinical and histopathologic analysis of 234 cases with evaluation of grading criteria. Cancer 1998;82(7):1217-1224

3 Ross L, Drazin D, Eboli P, Lekovic GP. Atypical tumors of the facial nerve: case series and review of the literature. Neurosurg Focus 2013;34(3):E2

4 Chenevert J, Barnes LE, Chiosea SI. Mucoepidermoid carcinoma: a five-decade journey. Virchows Arch 2011;458(2):133-140

5 Moore BA, Burkey BB, Netterville JL, Butcher RB II, Amedee RG. Surgical management of minor salivary gland neoplasms of the palate. Ochsner J 2008;8(4):172-180
6 Wilson JR, Kumar R, van Hille PT. Cerebellopontine angle metastasis of a parotid mucoepidermoid carcinoma arising from perineural invasion along the facial nerve. Br J Neurosurg 2012;26(3): 417-419

7 Yih WY, Kratochvil FJ, Stewart JC. Intraoral minor salivary gland neoplasms: review of 213 cases. J Oral Maxillofac Surg 2005;63(6): 805-810

8 Coca-Pelaz A, Rodrigo JP, Triantafyllou A, et al. Salivary mucoepidermoid carcinoma revisited. Eur Arch Otorhinolaryngol 2015; 272(4):799-819

9 Seifert G, Sobin LH. Histological typing of salivary gland tumors. World Health Organisation International Histological Classification of Tumors. 2nd ed. New York, NY: Springer-Verlag; 1991:20-41

10 Chen MM, Roman SA, Sosa JA, Judson BL. Histologic grade as prognostic indicator for mucoepidermoid carcinoma: a populationlevel analysis of 2400 patients. Head Neck 2014;36(2):158-163 